\title{
ON THE GENERALIZED OVERRELAXATION METHOD FOR OPERATION EQUATIONS ${ }^{1}$
}

\author{
W. V. PETRYSHYN ${ }^{2}$
}

1. Introduction. In [6] Householder, using Weissinger's identity, obtained the necessary and sufficient conditions for the convergence of the Gauss-Seidel method for the solution of finite matrix equations of the form $\left(D-S-S^{*}-F\right) u=f$. The same conditions were also obtained by Krein and Prozorovskaya [9] for an analog of the GaussSeidel method for the operator equations of the form $\left(D+S+S^{*}\right) u=f$ in a Hilbert space.

The purpose of this article is to extend the result of the above authors to the generalized overrelaxation method (go-method) for the solution of a wider class of operator equations $(D+S+Q) u=f$ in a Hilbert space investigated by the author [10].

Let us note that in case $H$ is finite-dimensional the overrelaxation method, in view of its practical success in the solution of finite algebraic systems arising in the numerical solution of partial differential equations, was previously extensively studied by a number of authors, in particular, by Frankel [4], Young [13], Arms, Gates and Zondek [2], Friedman [5], Varga [12], Keller [8], Kahan [7], Schechter [11], Albrecht [1] and others. ${ }^{3}$ Since the class of operator equations considered in this article includes, in particular, the Fredholm integral equations of the second kind with symmetric $\left(D=I, Q=S^{*}\right)$ and symmetrizable kernels and the finite matrix equations considered by most of the above authors our result will also at the same time unify and extend to our class of operators in Hilbert space the corresponding result of these authors. ${ }^{4}$

2. The identities. Let $H$ be a real or complex Hilbert space and $A$ a linear bounded operator in $H$ of the form

$$
A=D+S+Q \text {. }
$$

Let $S^{*}$ be the adjoint of $S$ and $\Omega$ a set of real positive numbers $\omega>0$

Received by the editors June 14, 1962 and, in revised form, September 6, 1962.

1 The work presented in this paper is partly supported by the AEC Computing and Applied Mathematics Center, Courant Institute of Mathematical Sciences, New York University, under Contract AT(30-1)-1480 with the U. S. Atomic Energy Commission, and the National Science Foundation.

2 Temporary member at the Courant Institute of Mathematical Sciences.

sor contributions of these and other authors to the development of the overrelaxation method see the comprehensive monograph by Forsythe and Wasow [3].

- The author is indebted to the referee for various suggestions concerning this paper. 
such that $\Omega$ and the operators $D, S$, and $Q$ have the property that there exists some linear symmetric and positive definite operator $K$ which commutes with $S$ and has the property that

(a) $(D u, K v)=(K u, D v)$ for all $u$ and $v$ in $H$,i.e., $D$ is $K$-symmetric,

(b) $G=G(\omega)=((2-\omega) / \omega) D+S^{*}-Q$ is $K$-symmetric and $K$-positive definite $(K$-p.d.), i.e., there exists a real number $\beta=\beta(\omega)>0$ such that for all $u \in H$ and $\omega \in \Omega$

$$
(G u, K u) \geqq \beta\|u\|^{2},
$$

(c) $(D+\omega S)$ has a bounded inverse defined on all of $H$ for $\omega \in \Omega$. Let us first observe that since $K$ is symmetric (a) implies that

$$
D^{*} K=K D
$$

while this together with (b) and the commutativity of $K$ and $S$ implies that

$$
Q^{*} K=K S-K S^{*}+K Q .
$$

Furthermore, (3) and (4) together imply that $A$ is $K$-symmetric, i.e., $A^{*} K=K A$.

In seeking an approximate solution $u_{n}$ of the equation

$$
A u=f, \quad f \in H,
$$

we shall apply the go-method with a constant relaxation factor $\omega$ in $\Omega$ defined by the scheme

$$
(D+\omega S) u_{n}=-\{(\omega-1) D+\omega Q\} u_{n-1}+\omega f,
$$

or equivalently by

$$
u_{n}=-T(\omega) u_{n-1}+g \text {, }
$$

where $u_{0}$ is an arbitrary initial approximation, $g=\omega(D+\omega S)^{-1} f$, and

$$
T(\omega)=(D+\omega S)^{-1}\{(\omega-1) D+\omega Q\}
$$

is the generalized overrelaxation operator.

Lemma 1. Let $D, S, Q, K$, and $\Omega$ satisfy the conditions (a), (b), and (c). Then for every $u$ and $v$ in $H$ the following identities are valid

$$
\begin{aligned}
& (K u, A v)=\sum_{i=0}^{n-1}\left(K T^{i}[I+T] u, G T^{i}[I+T] v\right)+R_{n}\{u, v\} \\
& \begin{array}{r}
|([D+\omega S] u, K u)|^{2}-|([(\omega-1) D+\omega Q] u, K u)|^{2} \\
=\omega^{2}(G u, K u)(A u, K u),
\end{array}
\end{aligned}
$$

where 
(11) $R_{n}\{u, v\}=\omega^{-1}\left(T^{n} u, K[D+\omega S] T^{n}(I+T) v\right), \quad n=1,2,3, \cdots$.

Proof. The proof of (9) and (10) requires some manipulation.

In establishing (9) we use the mathematical induction according to which we first verify its validity for $n=1$. If $u$ and $v$ are arbitrary elements in $H$, then by (8)

$$
[(\omega-1) D+\omega Q] u=(D+\omega S) T u
$$

or

$$
([(\omega-1) D+\omega Q] u, K v)=([D+\omega S] T u, K v)
$$

whence, using (3), we obtain

$$
\left(u,\left[(\omega-1) K D+\omega Q^{*} K\right] v\right)=\left(T u,\left[K D+\omega S^{*} K\right] v\right) .
$$

Adding and subtracting $(T u, K[(\omega-1) D+\omega Q] v)$ on the right and using the commutativity of $K$ with $S$ we get the identity

$\left(u,\left[(\omega-1) K D+\omega Q^{*} K\right] v\right)=(T u, \omega K G v)+(T u, K[(\omega-1) D+\omega Q] v)$

which, in view of (4) and (12), can be written as

$$
(K u,[D+\omega S] v)=([I+T] u, \omega K G v)+(T u, K[D+\omega S] T v) .
$$

Since, by (12), $\omega A u=D+\omega S+(\omega-1) D+\omega Q=(D+\omega S) u$ $+(D+\omega S) T u$ we obtain from it and (13)

$$
\begin{aligned}
(K u, \omega A v)= & (K u,(D+\omega S) v)+(K u,(D+\omega S) T v) \\
= & ((I+T) u, \omega K G v)+(T u, K(D+\omega S) T v) \\
& +((I+T) u, \omega K G T v)+(T u, K(D+\omega S) T \cdot T v) \\
= & \omega((I+T) u, K G(I+T) v)+(T u, K(D+\omega S) T(1+T) v) .
\end{aligned}
$$

On dividing by $\omega$ we get (9) for $n=1$.

Let us assume now that (9) is valid for $n=k$, i.e.,

$$
(K u, A v)=\sum_{i=0}^{k-1}\left(K T^{i}(I+T) u, G T^{i}(I+T) v\right)+R_{k}\{u, v\},
$$

and prove its validity also for $n=k+1$. This, however, follows from (9), (13), and (11) for if in (13) we replace $u$ by $T^{n} u$ and $v$ by $T^{n}(I+T) v$, then from (11) we get

$$
\begin{aligned}
R_{k}\{u, v\}= & \omega^{-1}\left((I+T) T^{n} u, \omega K G T^{n}(I+T) v\right) \\
& +\omega^{-1}\left(T^{n+1} u, K(D+\omega S) T^{n+1}(I+T) v\right) \\
= & \left((I+T) T^{n} u, K G T^{n}(I+T) v\right)+R_{k+1}\{u, v\} .
\end{aligned}
$$


Combining this with $(\overline{9})$ we obtain the validity of (9) for $n=k+1$ and thus the proof of (9) for all $n$.

To prove (10) note that since the complex conjugate of $[((D+\omega S) u, K u)]$ is $\left(\left(D+\omega S^{*}\right) u, K u\right)$ and

$$
\begin{aligned}
\omega^{2}(G u, K u)(A u, K u)= & \omega(G u, K u)((D+\omega S) u, K u) \\
& +\omega(G u, K u)(((\omega-1) D+\omega Q) u, K u)
\end{aligned}
$$

for every $u$ in $H$, then adding $\left(\left[D+\omega S^{*}-\omega G\right] u, K u\right) \cdot((D+\omega S) u, K u)$ and subtracting its equivalent $([(\omega-1) D+\omega Q] u, K u)$ $\cdot((D+\omega S) u, K u)$ on the right we get

$$
\begin{aligned}
& \omega^{2}(G u, K u)(A u, K u) \\
&=\left(\left(D+\omega S^{*}\right) u, K u\right) \cdot((D+\omega S) u, K u) \\
&-([(\omega-1) D+\omega Q] u, K u) \cdot([D+\omega S-\omega G] u, K u) \\
&=|((D+\omega S) u, K u)|^{2} \\
&-([(\omega-1) D+\omega Q] u, K u)\left((\omega-1) D+\omega S+\omega\left(Q-S^{*}\right) u, K u\right) .
\end{aligned}
$$

Since the complex conjugate of $[([(\omega-1) D+\omega Q] u, K u)]$ is $(K u,[(\omega-1) D+\omega Q] u)=\left(\left[(\omega-1) K D+\omega Q^{*} K\right] u, u\right)$ the relation $(4)$ implies that the last relation is exactly the identity (10). This completes the proof of Lemma 1.

REMARK. If we choose $K=I, Q=S^{*}$, and $\omega=1$, then the identity (9) reduces to the identity used in [9]. If in this case we, in addition, assume that $A$ is symmetric and positive definite, then from the identity (10) valid for all $u$ in $H$ we obtain the inequality used in [9].

In Lemma 2 below we will establish the relationship between the identity (10) and the important equality (21) in [10] and, in this particular case, also the relationship between (9) and (10).

LEMMA 2. If, in addition to conditions (a), (b) and (c), the spectrum $\sigma(T)$ of the operator $T(\omega)$ contains only eigenvalues $\mu$ of finite multiplicity with zero as its sole limit point, then the identity (9) reduces to the equality

$$
\begin{aligned}
(K u, A u)= & |1+\mu|^{2}(K u, G u) \sum_{i=0}^{n-1}|\mu|^{2 i} \\
& +\omega^{-1}|\mu|^{2 n}(1+\bar{\mu})(K u,[D+\omega S] u)
\end{aligned}
$$

and (10) to the equality (21) in [10] which, for $A$ of the form (1), can be written as

$$
(G u, K u)=\frac{1-|\mu|^{2}}{|1+\mu|^{2}}(A u, K u),
$$


where $\mu$ is an arbitrary eigenvalue of $T$ and $u \neq 0$ an eigenvector corresponding to $\mu$. Furthermore, if $A$ is also $K$-p.d. then for $n \rightarrow \infty$ the equality $\left(9^{\prime}\right)$ is identical with $\left(10^{\prime}\right)$.

Proof. The proof of $\left(9^{\prime}\right)$ follows directly from (9) with $v=u$ and the observation that the equality $T u=\mu u$ implies that $T^{i}(I+T) u$ $=\mu^{i}(1+\mu) u$ for $i=0,1,2, \cdots$, and $\left(K T^{i}(I+T) u, G T^{i}(I+T) u\right)$ $=|1+\mu|^{2}|\mu|^{2 i}(K u, G u)$.

To prove $\left(10^{\prime}\right)$ note that, in view of (8) and (12), Tu= $\mu u$ implies that

$$
[(\omega-1) D+\omega Q] u=(D+\omega S) T u=\mu(D+\omega S) u .
$$

Substituting this into the identity (10) we get

$$
\omega^{2}(G u, K u)(A u, K u)=\left[1-|\mu|^{2}\right]|((D+\omega S) u, K u)|^{2} .
$$

Noting that $T=(D+\omega S)^{-1}\{(\omega-1) D+\omega Q\}=\omega(D+\omega S)^{-1} A-I$ and hence that -1 is not an eigenvalue of $T$ we obtain the equality $(D+\omega S) u=[\omega /(1+\mu)] A u$ valid for all eigenvectors $u$ and corresponding eigenvalues $\mu$ of $T$. Now substituting $[\omega /(1+\mu)] A u$ for $(D+\omega S) u$ in (14) we obtain the desired equality $\left(10^{\prime}\right)$.

The validity of the last assertion in Lemma 2 follows from $\left(10^{\prime}\right)$ and $\left(9^{\prime}\right)$ for, since $G$ and $A$ are positive definite, the relation $\left(10^{\prime}\right)$ implies that $|\mu|<1$ and, therefore, passing to the limit in $\left(9^{\prime}\right)$ as $n \rightarrow \infty$ we obtain

$$
(K u, A u)=|1+\mu|^{2}(K u, G u) \sum_{i=0}^{\infty}|\mu|^{2 i}=\frac{|1+\mu|^{2}}{1-|\mu|^{2}}(K u, G u)
$$

which is the equality $\left(10^{\prime}\right)$.

3. The main theorem. Let us note that from (7) we find by induction that

$$
u_{n}=\sum_{i=0}^{n-1} T^{i} g+T^{n} u_{0}
$$

from which we see that the sequence of approximations $u_{n}$ converges if the series $\sum_{i=0}^{\infty} T^{i}$ converges. The latter converges if the spectrum $\sigma(T)$ of $T$ lies in the interior of the unit circle. We shall now prove the main

TheOREM. If $D, S, Q, K$, and $\Omega$ satisfy the conditions (a), (b), and (c), then the necessary and sufficient condition that the spectrum $\sigma(T)$ lie in the interior of the unit circle is that the operator $A$ be K-p.d.

Proof. The proof of the theorem is essentially based on the identities established in Lemma 1. 
Necessity. To prove it note that if $\sigma(T)$ lies in the interior of the unit circle, then it is not hard to see that $\left|R_{n}\{u, v\}\right| \rightarrow 0$ as $n \rightarrow \infty$ and the identity (9) implies that

$$
(K u, A v)=\sum_{i=0}^{\infty}\left(K T^{i}(I+T) u, G T^{i}(I+T) v\right)
$$

for all $u$ and $v$ in $H$. Thus, if in (16) we put $u=v$, then, in view of condition (b), $(A u, K u)$ is a convergent series of positive numbers. In particular, we have

$$
(K u, A u) \geqq(K(1+T) u, G(1+T) u) .
$$

Since $\lambda=-1$ does not belong to $\sigma(T)$, the last inequality shows that $A$ is $K$-p.d. and thus proves the necessity.

Sufficiency. To prove the sufficiency we must show that the assumption that $A$ is $K$-p.d., i.e., there exists an $\alpha>0$ such that for all $u$ in $H$

$$
(A u, K u) \geqq \alpha\|u\|^{2},
$$

implies that $(T-\lambda)$ is a continuously invertible operator for all $|\lambda| \geqq 1$, i.e., $R(T-\lambda)=H$ and there exists a $\theta>0$ such that $\|(T-\lambda) u\|$ $\geqq \theta\|u\|$ for all $u \in H$, where $R(T-\lambda)$ is the range of $(T-\lambda)$.

If $(T-\lambda)$ were not continuously invertible there would exist a sequence $\left\{u_{n}\right\},\left\|u_{n}\right\|=1$, such that $v_{n}=(T-\lambda) u_{n} \rightarrow 0$ as $n \rightarrow \infty$. Applying to $v_{n}$ the operator $(D+\omega S)$ we get $(D+\omega S) v_{n} \rightarrow 0$, i.e.,

$$
\{(\omega-1) D+\omega Q\} u_{n}-\lambda(D+\omega S) u_{n} \rightarrow 0
$$

as $n \rightarrow \infty$ or

$$
\left(\{(\omega-1) D+\omega Q\} u_{n}, K u_{n}\right)-\lambda\left(\{D+\omega S\} u_{n}, K u_{n}\right) \rightarrow 0 .
$$

Since we are dealing with bounded operators the last limiting relation implies that

(19) $\left.|\lambda|^{2} \mid(D+\omega S) u_{n}, K u_{n}\right)\left.\right|^{2}-\left|\left([(\omega-1) D+\omega Q] u_{n}, K u_{n}\right)\right|^{2} \rightarrow 0$

as $n \rightarrow \infty$. However, if $A$ is $K$-p.d. and $|\lambda| \geqq 1$, the limiting relation (19) contradicts the inequality derived from the identity (10) for, in view of (2) and (17), the identity (10) yields the inequality

$$
\left|\left((D+\omega S) u_{n}, K u_{n}\right)\right|^{2}-\left|\left([(\omega-1) D+\omega Q] u_{n}, K u_{n}\right)\right|^{2} \geqq \omega^{2} \beta \alpha .
$$

Thus, $(T-\lambda)^{-1}$ is a bounded operator defined on the closed subspace $R(T-\lambda) \subset H$. Moreover $R(T-\lambda)=H$ for otherwise there would exist an element $v$ in $H$ such that $((T-\lambda) u, v)=0$ or $(T u, v)=\lambda(u, v)$ 
for all $u$ in $H$. This can be written in the form $([(\omega-1) D+\omega Q] u$, $\left.\left(D^{*}+\omega S^{*}\right)^{-1} v\right)=\lambda(u, v)$. Since $R(K)=H$ there exists an element $w$ in $H$ such that $K w=\left(D^{*}+\omega S^{*}\right)^{-1} v$. If we choose now $u=w$, then the last equality becomes

$$
([(\omega-1) D+\omega Q] w, K w)=\lambda((D+\omega S) w, K w) .
$$

For $|\lambda| \geqq 1$ this equality contradicts the corresponding inequality (20) arising from the identity (10). This shows that $R(T-\lambda)=H$ and thus completes the proof of the theorem.

4. Special cases. (i) If we take $\omega=1$, then the theorem gives the necessary and sufficient conditions for the convergence of the generalized Gauss-Seidel method, which for the case when $K=I$ and $Q=S^{*}$ were given in [9].

(ii) If we change the signs of $S$ and $Q$ and let $\omega=1, K=I$, and $Q=S^{*}+F$, where $F$ is symmetric, the theorem reduces to the result proved in [6] for the case when the operators are finite matrices.

(iii) If $D=I$ and $Q=S^{*}$, then the theorem and the go-method are applicable to the operator equations of the form $\left(I-S-S^{*}\right) u=f$ to which are reducible, for example, the Fredholm integral equations of the second kind with symmetric or symmetrizable kernels and matrix equations in which $D$ is usually the matrix composed of diagonal terms, $S$ is a lower triangular, and $S^{*}$ an upper triangular matrix. Let us note at the end that for the last two important classes of operator equations the set $\Omega=\{\omega, 0<\omega<2\}$.

(iv) Theorem 1 in [10] is a special and a more restrictive case of the present theorem.

\section{REFERENCES}

1. J. Albrecht, Fehlerabschatzungen bei Relaxationsverfahren sur numerischen Auf. lösung linearen Gleichungssystemen, Numer. Math. (3) 3 (1961), 188-201.

2. R. I. Arms, L. D. Gates and B. Zondek, A method of block iteration, J. Soc. Indust. Appl. Math. 4 (1956), 220-229.

3. G. E. Forsythe and W. Wasow, Finite-difference methods for partial differential equations, Wiley, New York, 1960.

4. S. P. Frankel, Convergence rates of iterative treatments of partial differential equations, Math. Comp. 4 (1950), 65-75.

5. B. Friedman, The iterative solution of elliptic difference equations, AEC Research and Development Report NYO-7698, New York University, New York, 1957.

6. A. S. Householder, On the convergence of matrix iterations, J. Assoc. Comput. Mach. 3 (1956), 314-324.

7. W. Kahan, The rate of convergence of the extrapolated Gauss-Seidel iterations (abstract), J. Assoc. Comput. Mach. 4 (1957), 521-522.

8. H. B. Keller, On some iterative methods for solving elliptic difference equations, Quart. Appl. Math. 16 (1958), 209-226. 
9. S. G. Krein, and O. I. Prozorovskaya, An analogue of Seidel's method for operator equations, Voronez. Gos. Univ., Trudy Sem. Functional. Anal. 5 (1957), 35-38.

10. W. V. Petryshyn, The generalized overrelaxation method for the approximate solution of operator equations in Hilbert space, J. Soc. Indust. Appl. Math. 10 (1962), 675-690.

11. S. Schechter, Relaxation methods for linear equations, Comm. Pure Appl. Math. 12 (1959), 313-335.

12. R. S. Varga, A comparison of the successive overrelaxation method and semiiterative methods using Chebyshev polynomials, J. Soc. Indust. Appl. Math. 5 (1957), 39-46.

13. D. Young, Iterative methods for solving partial difference equations of elliptic type, Trans. Amer. Math. Soc. 76 (1954), 91-111.

NeW YoRK UNIVERSITY

\section{NOTE ON A NONLINEAR VOLTERRA EQUATION}

\section{J. J. LEVIN ${ }^{1}$ AND J. A. NOHEL 2}

1. Introduction. We investigate the solutions of

$$
x^{\prime}(t)=-\int_{0}^{t} a(t-\tau) g(x(\tau)) d \tau \quad\left(,=\frac{d}{d t}\right)
$$

as $t \rightarrow \infty$, where $a(t)$ is completely monotonic on $0 \leqq t<\infty$ and where $g(x)$ is a (nonlinear) spring. Under this hypothesis, (1.1) was shown in [2] to be relevant to certain physical applications and results were obtained there for the linear case $g(x) \equiv x$. (If $a(t) \equiv a(0)$, then (1.1) reduces to the nonlinear oscillator $x^{\prime \prime}+a(0) g(x)=0$.) Equation (1.1) was studied in [1] under less hypothesis on $a(t)$. However, while the result is weaker than that of [1], the present approach draws together such different notions of positivity as Liapounov functions, completely monotonic functions, and kernels of positive type. It also provides a new Liapounov function for (1.1). Specifically, we prove the

ThEOREM. Let $a(t)$ and $g(x)$ satisfy

$$
\begin{array}{r}
a(t) \in C[0, \infty),(-1)^{k} a^{(k)}(t) \geqq 0(0<t<\infty ; k=0,1,2, \cdots), \\
g(x) \in C(-\infty, \infty), x g(x)>0(x \neq 0), G(x)=\int_{0}^{x} g(\xi) d \xi \rightarrow \infty \\
(|x| \rightarrow \infty) .
\end{array}
$$
1962.

Presented to the Society, January 24,1963 ; received by the editors September 6 ,

${ }^{1}$ MIT Lincoln Laboratory. Operated with support from the U. S. Army, Navy, and Air Force.

2 Partially supported by the National Science Foundation (G-19925). 